\title{
Streamer studies in Resistive Plate Chambers.
}

\author{
A. Paoloni, ${ }^{1}$ A. Mengucci, M. Spinetti, M. Ventura, and L. Votano. \\ INFN-LNF, \\ via E. Fermi 4000044 Frascati (RM), Italy. \\ E-mail: alessandro.paoloni@lnf.infn.it
}

\begin{abstract}
The present paper is meant as an update of the presentation given in a previous Resistive Plate Chamber (RPC) workshop, aimed at finding an eco-friendly gas mixture for streamer operation of RPCs. Indeed the streamer working regime is still suitable for building large RPC systems dedicated to low rate applications, such as cosmic ray and neutrino physics. In addition to other studies about gas mixtures for streamer mode operation, in this paper the replacement of R134a with $\mathrm{CF}_{4}$, a gas widely used in other gaseous detectors, has been investigated.

The effect of the gas gap thickness on the discharge quenching has also been studied; this is an important check because thin gas gaps of $1 \mathrm{~mm}$, one half of the typical used value, have been introduced for high rate applications.

Finally preliminar results about the streamer formation timing are also reported.
\end{abstract}

KeYwords: Gaseous detectors, Resistive plate chambers, Electrical discharge in gases.

\footnotetext{
${ }^{1}$ Corresponding author.
} 


\section{Contents}

1 Introduction 1

2 Streamer operation of RPCs filled with gas mixtures containing $\mathbf{C F}_{4} \quad 1$

3 Streamer operation of RPCs with $1 \mathbf{m m}$ gas gap and electrode thickness 3

4 Streamer formation time studies $\quad 3$

5 Conclusions 5

\section{Introduction}

In recent years, in view of upgrades to some of the LHC experiments (ATLAS, CMS and ALICE), most of the R\&D activity on Resistive Plate Chambers (RPCs) has been devoted to avalanche operation (see for instance [1]). Nevertheless, low rate applications, such as neutrino [2] or astroparticle physics [3], could still use RPCs operated in streamer mode, profiting of electronics simplicity (no amplification needed) and of the existence of safe and eco-friendly gas mixtures made of argon (Ar) and tetrafluoropropene (HFO-1234ze) [4].

In [4] results of studies about mixtures containing carbon dioxide $\left(\mathrm{CO}_{2}\right)$, nitrogen $\left(\mathrm{N}_{2}\right)$, helium $(\mathrm{He})$ and tetrafluoromethane $\left(\mathrm{CF}_{4}\right)$, a gas widely used for other gaseous detectors (see [5] for instance), were also reported. In section 2 results about additional investigations performed on the use of tetrafluoromethane $\left(\mathrm{CF}_{4}\right)$ are reported. The effects of the gas gap thickness on the streamer discharge are also discussed in section 3; in particular the performance of RPCs with $1 \mathrm{~mm}$ gas gap and $1 \mathrm{~mm}$ electrode thickness, a possible new standard in the detector production for LHC experiments, is shown. Finally studies about streamer timing are reported in section 4: these measurements could be exploited to disentangle between different models of streamer formation.

\section{Streamer operation of RPCs filled with gas mixtures containing $\mathbf{C F}_{4}$}

Typical gas mixtures for streamer mode operation of RPCs are composed by Ar and one or more gases, known as "quenchers", needed to absorb UV photons, produced by Ar de-excitation, without re-emission. Good quenchers are isobutane $\left(\mathrm{i}-\mathrm{C}_{4} \mathrm{H}_{10}\right)$, whose percentage is limited to few $\%$ because of safety requirements on flammability, tetrafluoroethane (R134a) and tetrafluoropropene (HFO-1234ze, HFO-1234yf). The addition of sulfur hexafluoride $\left(\mathrm{SF}_{6}\right)$ permits the operation in streamer with a strongly decreased charge. While in [4] $\mathrm{CF}_{4}$ was investigated as a substitute for $\mathrm{SF}_{6}$, here it is investigated as a replacement for R134a.

The studies described in this section have been performed, using the same set-up, used for gas mixture tests, described in [4]. Cosmic rays crossing the detector under study, a $60 \times 70 \mathrm{~cm}^{2}$ wide 

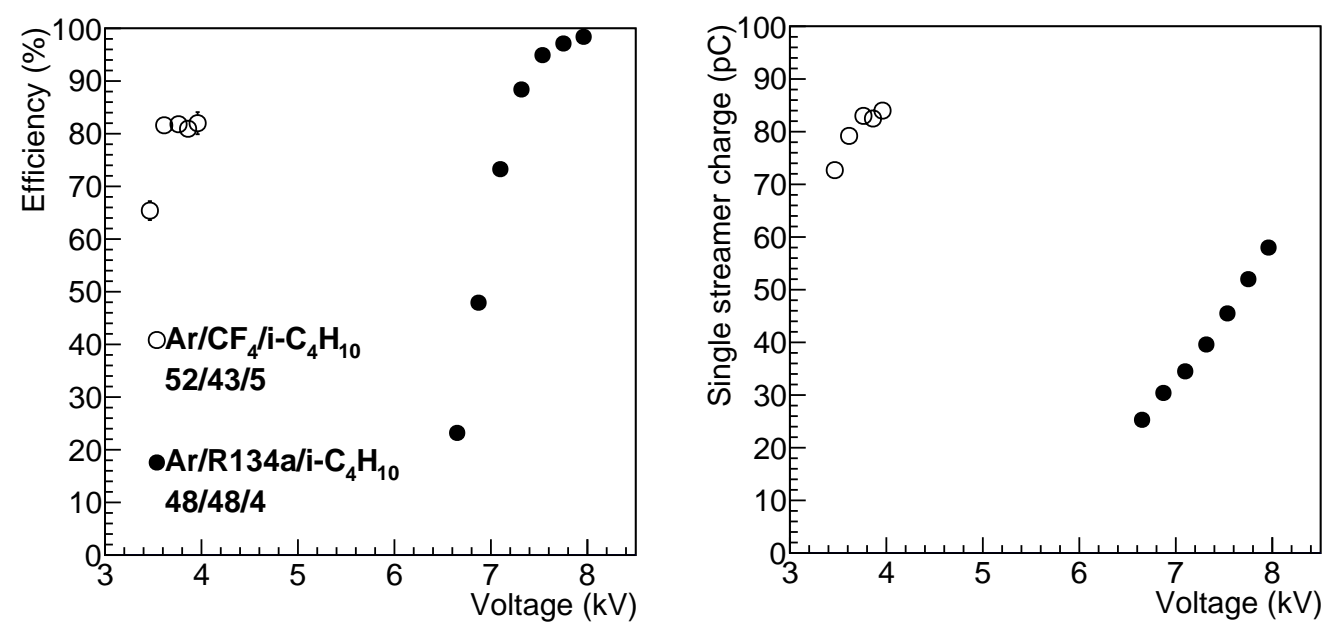

Figure 1. Efficiency and single streamer charge, as a function of the operating voltage, for the gas mixture $\mathrm{Ar} / \mathrm{CF}_{4} / \mathrm{i}-\mathrm{C}_{4} \mathrm{H}_{10}$ in the volume ratios $52 / 43 / 5$ (empty circles). As a reference, the same measurements performed with the gas mixture $\mathrm{Ar} / \mathrm{R} 134 \mathrm{a} / \mathrm{i}-\mathrm{C}_{4} \mathrm{H}_{10}$ in the volume ratios 48/48/4 (full circles) are also reported.

RPC, have been triggered by means of scintillators. The gas gap and the two bakelite electrodes are all $2 \mathrm{~mm}$ thick. Streamer discharges in the gas gap induce opposite polarity signals on both sides of the chamber. Those signals are picked up by means of $3.5 \mathrm{~cm}$ wide copper strips, faced to the external faces of the electrodes. The strips are terminated, on the read-out side, on $110 \Omega$, and on the opposite side on their characteristic impedance, $25 \Omega$. Signals from the read-out strips are digitized at $5 \mathrm{GS} / \mathrm{s}$ and acquired.

In figure 1 the efficiency and the single streamer charge are shown as a function of the operating voltage for a gas mixture composed of $\mathrm{Ar}, \mathrm{CF}_{4}$ and $\mathrm{i}-\mathrm{C}_{4} \mathrm{H}_{10}$ in the volume ratios 52/43/5. As a reference, the same measurements for a gas mixture composed of $\mathrm{Ar}, \mathrm{R} 134 \mathrm{a}$ and $\mathrm{i}-\mathrm{C}_{4} \mathrm{H}_{10}$ in the volume ratios 48/48/4, are shown. The concentrations of $\mathrm{Ar}$ and $\mathrm{i}-\mathrm{C}_{4} \mathrm{H}_{10}$ are similar and therefore the different performances of the two different mixtures can be directly ascribed to the replacement of $\mathrm{R} 134 \mathrm{a}$ by $\mathrm{CF}_{4}$. The detector, flushed with the mixture containing $\mathrm{CF}_{4}$, reaches the maximum efficiency at $3.5 \mathrm{kV}$, more than $4 \mathrm{kV}$ below the reference mixture with $\mathrm{R} 134 \mathrm{a}$; the efficiency plateau value is around $80 \%$ with a much higher streamer charge. This behavior has been observed flushing RPCs with $2 \mathrm{~mm}$ gas gap with mixtures containing small percentages of quencher gases. As an example, a similar trend was observed in studies about ternary mixtures made by Ar, R134a and i- $\mathrm{C}_{4} \mathrm{H}_{10}$, decreasing the $\mathrm{R} 134$ a percentage below $20 \%$ at fixed $4 \% \mathrm{i}-\mathrm{C}_{4} \mathrm{H}_{10}$ concentration [6].

In figure 2 typical single streamer wave-forms are also shown for the two considered gas mixtures. The mixture with $\mathrm{CF}_{4}$ has a higher integrated charge ( $85 \mathrm{vs} 60 \mathrm{pC}$ ), resulting from a lower amplitude ( $150 \mathrm{vs} 350 \mathrm{mV} / 110 \Omega$ ) and a higher full-width-at-half-maximum (45 vs $12 \mathrm{~ns}$ ). It has also a higher rise-time (4 vs $2.5 \mathrm{~ns}$ from $10 \%$ to $90 \%$ of the signal amplitude).

In conclusion $\mathrm{CF}_{4}$ is not advisable as a quencher component inside gas mixtures for streamer operation of RPCs, because much better performances, in terms of efficiency and signal charge, can be obtained with R134a. 

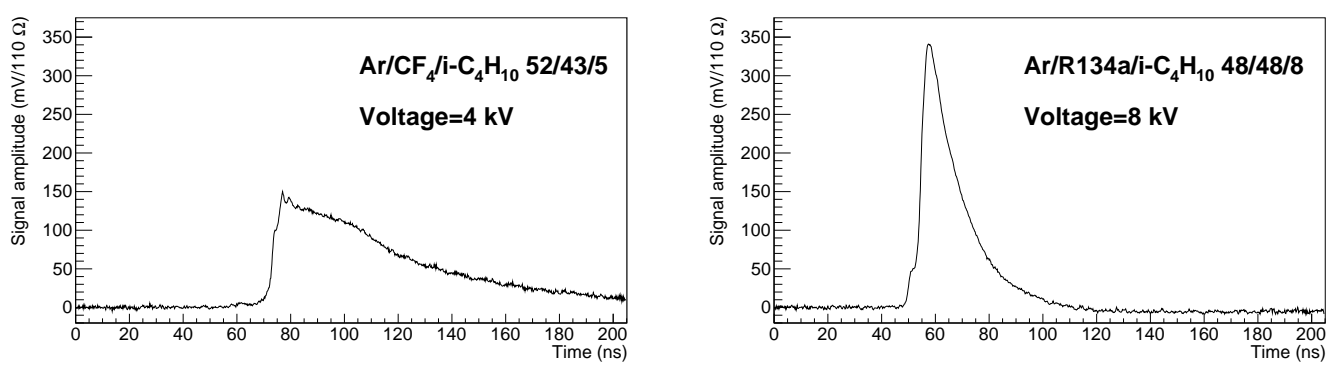

Figure 2. Typical wave-forms acquired with the gas mixtures $\mathrm{Ar} / \mathrm{CF}_{4} / \mathrm{i}-\mathrm{C}_{4} \mathrm{H}_{10}$ in the volume ratios $52 / 43 / 5$ at $4 \mathrm{kV}$ (left) and $\mathrm{Ar} / \mathrm{R} 134 \mathrm{a} / \mathrm{i}-\mathrm{C}_{4} \mathrm{H}_{10}$ in the volume ratios $48 / 48 / 4$ at $8 \mathrm{kV}$ operating voltage (right).

\section{Streamer operation of RPCs with $1 \mathrm{~mm}$ gas gap and electrode thickness}

Future upgrades of LHC RPC systems, designed for high rate operation in avalanche mode, are defining a new "standard" for the production of bakelite RPC gaps. Instead of $2 \mathrm{~mm}$ gas gaps with $2 \mathrm{~mm}$ electrode thickness, used so far in large systems, profiting of the lower charge released in the gas, of the better time resolution and of the lower operating voltage, future detectors will have thinner gas gaps.

A study about streamer mode operation of a $10 \times 10 \mathrm{~cm}^{2}$ wide RPC, with $1 \mathrm{~mm}$ gas gap and 1 mm bakelite electrodes thickness, is reported in [7]. The detector was read-out by means of a single copper pad and flushed with gas mixtures made of Ar, HFO-1234ze and $\mathrm{SF}_{6}$. Decreasing the gas gap, streamers are faster and with lower charge. The time resolution also improves.

In figure 3 the measured efficiency values are shown as a function of the operating voltage. With the considered gas mixtures, smaller plateau values are obtained using the RPC with $1 \mathrm{~mm}$ gas gap.

The multi-streamer probability has been measured as a function of the efficiency, checking by eye on a subsample of acquired wave-forms, the presence of after-pulsing and/or of streamers with an abnormal amplitude (twice that of the standard ones). The corresponding results are also shown in figure 3. It is evident that the multi-streamer probability, at fixed gas gap value, increases for decreasing HFO-1234ze (the quencher gas) concentrations. For a fixed gas mixture, the multistreamer probability strongly increases, at high efficiency, replacing the $2 \mathrm{~mm}$ gas gap RPC with the $1 \mathrm{~mm}$ gap one. In the latter case, above $90 \%$ efficiency, with all the considered gas mixtures, the multi-streamer probability is greater than $50 \%$. It is therefore preferable not to use $1 \mathrm{~mm}$ gas gap RPCs in streamer mode.

\section{Streamer formation time studies}

The time interval between avalanche precursor and streamer has been measured on a $10 \times 10 \mathrm{~cm}^{2}$ wide RPC with $2 \mathrm{~mm}$ gas gap flushed with a gas mixture composed of Ar (89\%) and HFO-1234ze $(11 \%)$ with and without the addition of $0.3 \% \mathrm{SF}_{6}$. The test has been performed using cosmic rays with a set-up similar to that used for the studies of the previous section. It is worth mentioning that the identification of the avalanche precursor has not been always possible. Two acquired waveforms are shown in figure 4 as an example: in the waveform shown in the left panel, the avalanche 

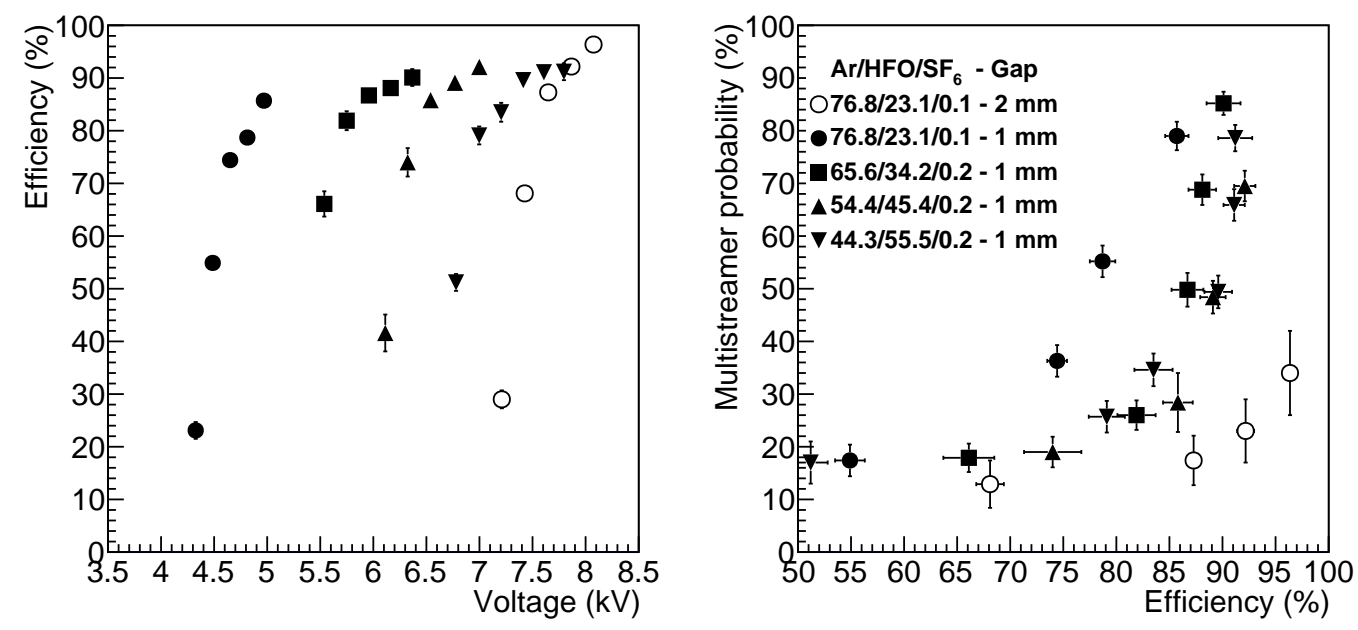

Figure 3. Efficiency and multi-streamer probability for streamer operation of RPCs with gas mixtures made of argon, HFO-1234ze and $\mathrm{SF}_{6}$. Both geometries with $2 \mathrm{~mm}$ (empty markers) and $1 \mathrm{~mm}$ (full markers) have been considered.
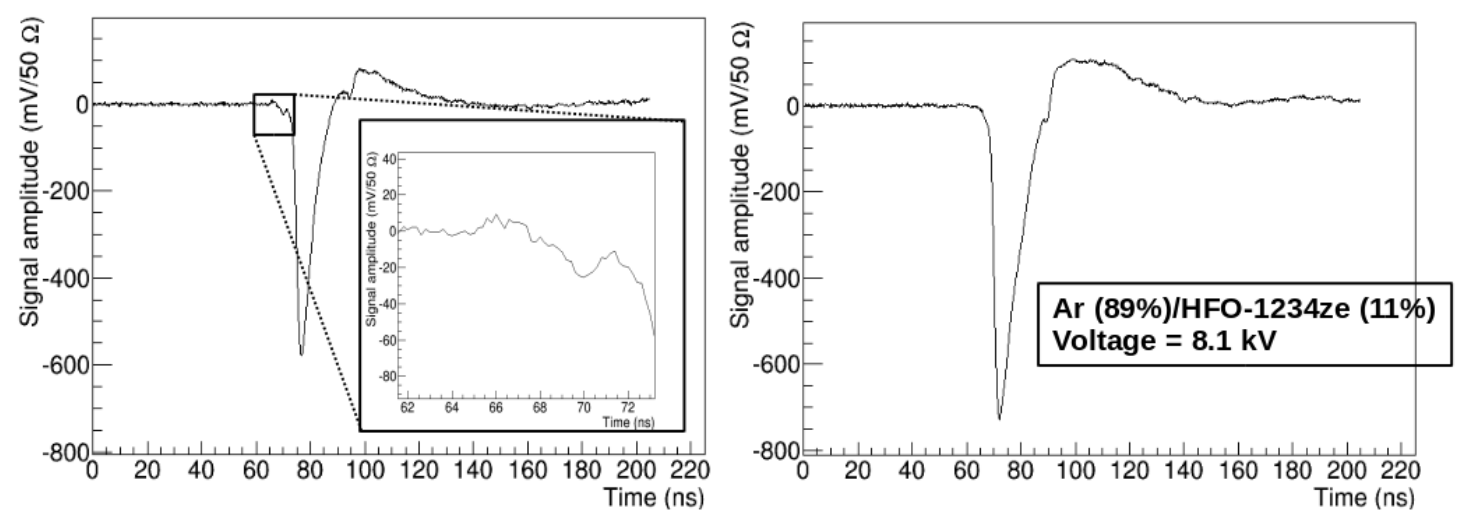

Figure 4. Examples of streamer wave-forms acquired at full efficiency for an RPC detector flushed with a gas mixture composed of $\operatorname{Ar}(89 \%)$ and HFO-1234ze (11\%). In the left plot, the avalanche precursor is well separated from the streamer and magnified in the insert.

precursor is clearly visible and separated from the following streamer, while in the waveform of the right panel it is not. The streamer delay has been measured only in the first case, as the time difference between the minima of the two signals.

With the considered gas mixtures, the detector reaches full efficiency around $8 \mathrm{kV}$. Two examples of the streamer delay distribution, as defined above, are shown in figure 5 at $8.15 \mathrm{kV}$ (for the mixture containing $\mathrm{SF}_{6}$ ) and $8.1 \mathrm{kV}$ (for the mixture without $\mathrm{SF}_{6}$ ) operating voltages, respectively. The corresponding average values are $(8.1 \pm 0.3) \mathrm{ns}$ and $(8.3 \pm 0.2) \mathrm{ns}$. The distributions are asymmetrical, with the average value greater than the most probable one and queues reaching also values few ns above the average value.

Other informations about how the streamer timing depends on the gas mixture, have been 

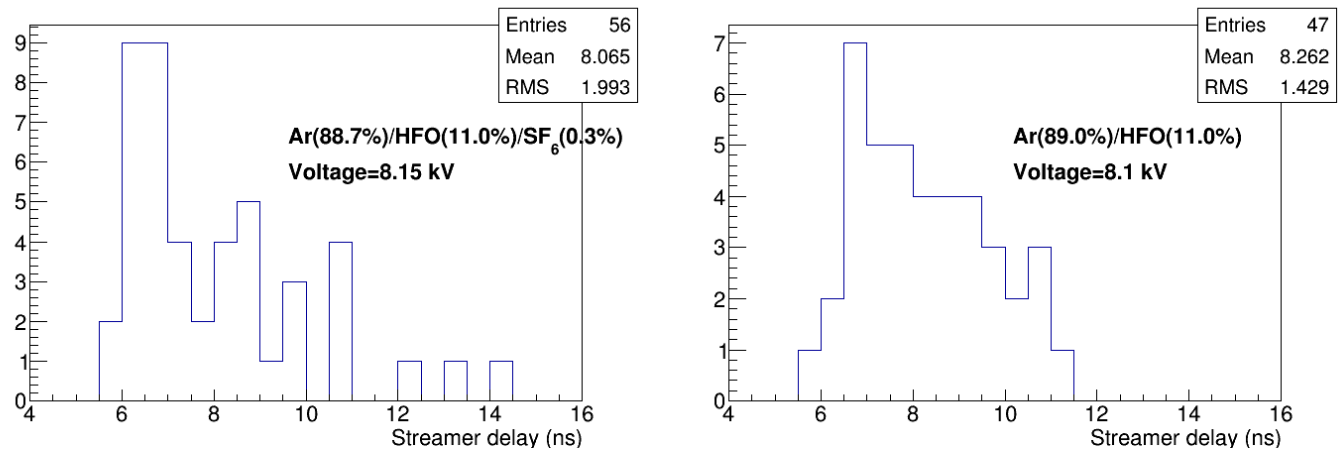

Figure 5. Examples of streamer delay distributions measured, with respect to the avalanche precursor, at full efficiency, for a $2 \mathrm{~mm}$ gap RPC flushed with a gas mixture composed of $\mathrm{Ar}(89 \%)$ and HFO-1234ze(11\%) with (left plot) and without (right plot) $\mathrm{SF}_{6}$ addition.
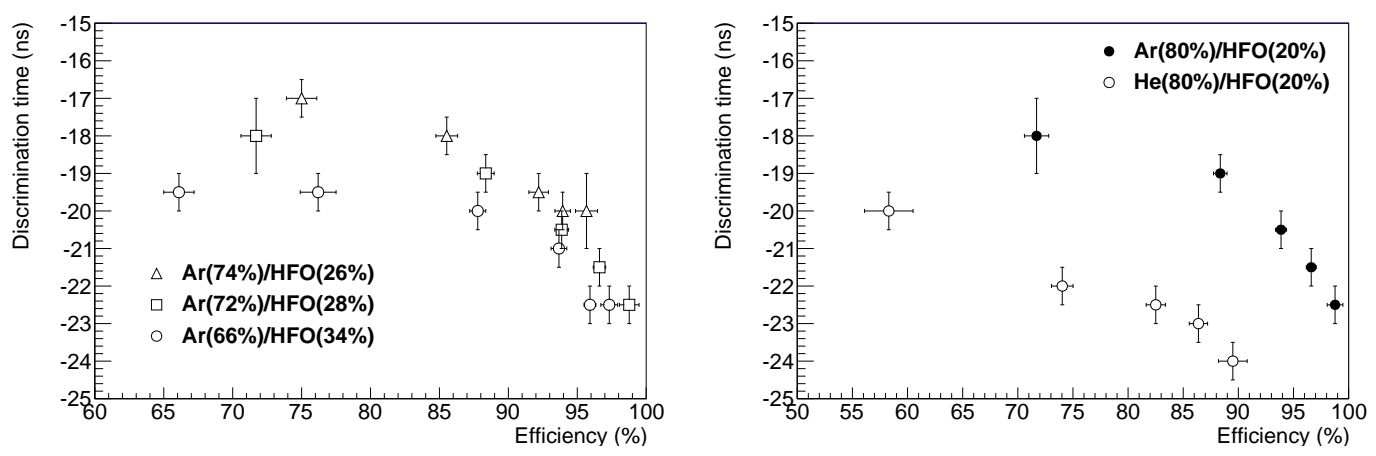

Figure 6. Streamer discrimination time vs efficiency for different binary mixtures made of Ar and HFO$1234 y f$ (left plot). In the right plot a comparison is done at fixed HFO-1234ze concentration between Ar and He.

obtained profiting of the data collected for the extensive gas mixture studies presented in [4]. In that analysis, signals, acquired from $3.5 \mathrm{~cm}$ wide copper strips, were discriminated at $50 \mathrm{mV}$ on a $110 \Omega$ load and the corresponding time registered. The discrimination time of the signal from one of the trigger scintillators was used as a reference and subtracted. Because of the delays due to the photomultiplier and to the cabling, the difference between the streamer discrimination and the reference scintillator times are negative. In the left panel of figure 6 , the streamer discrimination times are compared as a function of the efficiency for different HFO-1234yf (the quencher component) concentrations in Argon based gas mixtures. A similar study is performed in the right panel at fixed HFO-1234ze concentration, replacing Ar with Helium (He).

From the plots it is evident that at full efficiency the streamer time is almost independent from the HFO-1234yf percentage, while the use of He, instead of Ar, leads to a faster streamer development.

\section{Conclusions}

In this paper different aspects of streamer operation of RPCs are discussed. 
Concerning gas mixtures studies, tetrafluoromethane has been tested in mixtures as a replacement for quencher gases together with argon and isobutane: results similar to mixtures with insufficient quenching (low operating voltage and efficiency, large signals charge and width) have been observed, making tetrafluoromethane use not advisable in RPCs.

New chambers, built according to the new geometry introduced for avalanche operation at high rate, with $1 \mathrm{~mm}$ gas gap and $1 \mathrm{~mm}$ electrode thickness, have been tested in streamer mode. A strong increase of the multi-streamer probability is observed, suggesting to keep the old geometry $(2 \mathrm{~mm}$ gas gap and electrode thickness) for streamer mode operation.

Using gas mixtures made of argon and tetrafluoropropene (HFO-1234yf and HFO-1234ze), streamer timing studies have been performed. The time separation between avalanche and streamer has been found to be around $8 \mathrm{~ns}$ on average at about $8 \mathrm{kV}$ operating voltage, with distribution tails extending to values few ns above. In addition, a faster streamer formation has been observed, for a fixed tetrafluoropropene concentration, replacing argon with helium in the gas mixture.

\section{References}

[1] G. Aielli et al., Improving the RPC rate capbility, JINST 11 (2016) P07014.

[2] The OPERA Collaboration, The OPERA experiment in the CERN to Gran Sasso neutrino beam, JINST 4 (2009) P04018, pag.28.

[3] ARGO Collaboration, Astroparticle Physics with ARGO, Proposal (1996).

[4] A. Paoloni, A. Longhin, A. Mengucci, F. Pupilli, M. Ventura, Gas mixture studies for streamer operated Resistive Plate Chambers, JINST, 11 (2016) C06001.

[5] G. Bencivenni et al., A triple GEM detector with pad readout for high rate charged particle triggering, NIMA, 488 (2002) 493.

[6] A. Paoloni et al., Streamer studies in Resistive Plate Chambers, NIMA, 640 (2011) 76.

[7] A. Paoloni, A. Mengucci, M. Spinetti, M. Ventura and L. Votano, Gas gap studies about streamer operated RPCs, JINST, 12 (2017) P05020. 